$5-1-2003$

\title{
The Effect of Ultra Low Dose Epidural Analgesia on Newborn Breastfeeding Behaviors
}

Sharon Radzyminski

Cleveland State University, s.radzyminski@csuohio.edu

Follow this and additional works at: https://engagedscholarship.csuohio.edu/nurs_facpub

Part of the Nursing Commons

How does access to this work benefit you? Let us know!

Publisher's Statement

This is the accepted version of the following article: Radzyminski, S. (2003). The effect of ultra low dose epidural analgesia on newborn breastfeeding behaviors. Journal of Obstetric, Gynecologic, \& Neonatal Nursing, 32(3), 322-331. doi:10.1177/0884217503253440, which has been published in final form at http://onlinelibrary.wiley.com/doi/10.1177/0884217503253440/ epdf

\section{Recommended Citation}

Radzyminski, Sharon, "The Effect of Ultra Low Dose Epidural Analgesia on Newborn Breastfeeding Behaviors" (2003). Nursing Faculty Publications. 45.

https://engagedscholarship.csuohio.edu/nurs_facpub/45

This Article is brought to you for free and open access by the School of Nursing at EngagedScholarship@CSU. It has been accepted for inclusion in Nursing Faculty Publications by an authorized administrator of EngagedScholarship@CSU. For more information, please contact library.es@csuohio.edu. 


\title{
The Effect of Ultra Low Dose Epidural Analgesia on Newborn Breastfeeding Behaviors
}

\author{
Sharon Radzyminski
}

Objective: To determine whether a difference in breastfeeding behaviors could be observed between newborns whose mothers received epidural analgesia for labor pain relief and those newborns whose mothers received no pain medication in labor.

Design: There were two groups of neonates in this study. One group was born to mothers who received epidural analgesia, and one group was born to mothers who received no pain medication for labor. Both groups were observed for initial breastfeeding behaviors using the Premature Infant Breastfeeding Behavior Scale following birth and at 24 hours. Central nervous system functioning in the newborn was measured with the Neurologic and Adaptive Capacity Score at 2 and 24 hours of age. Ohio.

Setting: A large tertiary hospital in northeast

Participants: Fifty-six breastfeeding mothernewborn dyads. All mothers were healthy multiparae who gave birth vaginally to normal, full-term, healthy newborns.

Main Outcome Measures: Newborns were observed for rooting, latch on, sucking, swallowing, activity state, and neurobehavior.

Results: There were no statistically significant differences in breastfeeding behaviors at birth or at 24 hours of age.

Conclusion: A possible cause for the lack of significant results may have been the ultra low dose of bupivacaine and fentanyl used in this sample. JOGNN, 32, 322-331; 2003. DOl: 10.1177/ 0884217503253440
Keywords: Analgesia -Anesthesia - Breastfeeding-Epidural-Newborn feeding

Although breastfeeding a newborn has been shown to improve his or her health, many women are unsuccessful in their attempts. A number of factors have been identified for lack of success in breastfeeding, including sore nipples (Chapman, Macey, Keegan, Borum, \& Bennett, 1985; Frantz \& Fleiss, 1980), engorgement (Chapman et al., 1985; O'Leary, Kopsell, \& Haller, 1997), milk insufficiency (Chapman et al., 1985), poor newborn weight gain (O'Leary et al., 1997), difficulty of the newborn in latching onto the breast or sucking (Hughes, Townsend, \& Branum, 1988; Lothian, 1995; Righard \& Alade, 1992, Walker, 1997), and a crying, discontented baby (Hughes et al., 1988; Vandiver, 1997). In addition, the literature has identified the use of pain medication by the mother during labor, specifically epidural analgesia, as a potential barrier to breastfeeding success (Crowell, Hill, \& Humenick, 1994; Kuhnert, Linn, \& Kuhnert, 1985; Righard \& Alade, 1992; Sapkoski, Lester, Ostheimer, \& Brazelton, 1992).

The reason epidural analgesia could pose a potential barrier to breastfeeding is the popular use of narcotics in combination with local anesthetics to reduce maternal pain during labor. This drug combination could cross the placenta and depress crucial neonate reflexes needed for feeding, such as rooting, 
sucking, or swallowing. In addition, neonate tone may be depressed, which could influence the newborn's ability to latch on or remain latched onto the breast. The purpose of this study was to determine whether a difference in breastfeeding behaviors could be detected between newborns whose mothers received epidural analgesia for labor pain relief and those whose mothers received no pain medication for labor.

Bupivacaine (Marcaine, Sanofi Winthrop Pharmaceuticals, Morrisville, PA) is the most commonly used local anesthetic for epidural analgesia given to laboring women. When reports began to appear in the literature related to the use of bupivacaine in epidural analgesia for labor pain management, the total dose reported was typically $130 \mathrm{mg}$ or higher. At this dose, there were isolated reports of decreased neonatal muscle tone (Weiner, Hogg, \& Rosen, 1979) and decreased neonate sucking for 24 hours after delivery (Kileff, James, Dewan, \& Floyd, 1984). Other reports, however, indicated no difference in neonatal behavior (Amano, Nishijima, \& Arai, 1985; Kangas-Saarela, Jouppila, \& Jouppila, 1987; Loftus, Hill, \& Cohen, 1995; Murray, Dolby, Nation, \& Thomas, 1981; Rosenblatt et al., 1981; Scherer \& Holzgreve, 1995) as measured by Apgar scores (Belsey et al., 1981; Blackbill, Kane, Manniello, \& Abramson, 1974), acidbase reports (Abboud, Khoo, Miller, Doan, \& Henriksen, 1982; Eddleston et al., 1992; Jouppila \& Hollmen, 1976; Okutomi, Mochizuki, Asmano, \& Hoka, 2000; Scherer \& Holzgreve, 1995; Swanstrom \& Bratteby, 1981; Zador \& Nilsson, 1974), or neurological examination findings on the Brazelton (Brazelton, 1961; Sapkoski et al., 1992; Tronick, Wise, \& Ats, 1976), Early Neonatal Neurobehavioral Scale (Corke, 1977; Scanlon, Ostheimer, Lurie, \& Briwb, 1976), or the Neurologic and Adaptive Capacity Score (Amid-Tison, Barrier, \& Shnider, 1982; Hoyt \& Youngstrom, 1990).

It should be noted, however, that much of the research related to the effects of epidural analgesia on the neonate was conducted in the late 1970s through the late 1980s, when epidural analgesia was becoming increasingly popular. During this time period, the type of drug, dosage, and method of epidural administration varied considerably (Bonica \& MacDonald, 1995). It is interesting to note that even with the frequent changes in the administration of epidural analgesia for pain relief in labor, a common finding was the Lack of negative outcomes for the neonate. This was in contrast with information related to the use of meperidine in labor and the depressive effects that were being demonstrated in the neonate during this same time period (Hodgkinson \& Husain, 1982). In some instances, the literature became confusing when authors inferred that the results reported in studies of meperidine also applied to the use of epidural analgesia. The Lack of reported negative outcomes for the neonate as a result of the use of epidural analgesia for almost 30 years has consequently resulted in few recent studies that have addressed this issue. Unfortunately, however, neonate feeding was not a variable that was considered when negative outcomes on the neonate were investigated. This has left a large gap in the literature, especially in relation to the effect on breastfeeding newborns.

Fentanyl (Sublimaze, Abbott Laboratories, Chicago [Fentanyl citrate inj. $50 \mathrm{mcg} / \mathrm{ml}$ ]) is a narcotic commonly used in combination with bupivacaine for epidural analgesia for labor. Neonatal depression has been reported following doses of $600 \mathrm{mcg}$ (Carrie, O'Sullivan, \& Seegobin, 1981), but not at doses lower than $100 \mathrm{mcg}$ (Bader et al., 1995). These studies, however, did not include the newborn's ability to feed in their evaluation of neonatal depression.

Research findings that do take into consideration the newborn's ability to feed are divided on how great an effect, if any, epidural analgesia has on breastfeeding. This is not surprising considering the variety of drugs available for epidural analgesia that can be given in multiple combinations, dosages, and methods of administration. In addition, studies have been difficult to interpret because other variables suspected to influence breastfeeding, such as supplementation and time of initiation of feeding, were not well controlled for in the study design; measurements of drug accumulations have not been reported; there has been a lack of control subjects; and the measurement tools used to evaluate breastfeeding have been in the early stages of development. In light of these difficulties, there have been attempts to investigate this phenomenon.

Reports in the literature date back to the 1980s as researchers began to investigate the effect of epidural analgesia on neonatal feeding behavior (Hodgkinson, Blatt, \& Wang, 1978; Kuhnert et al., 1985). These early reports used neurological examinations as the measurement tool, but they contained only minimal evaluation of the newborn's ability to suck. In addition, sucking or feeding was usually measured with bottle-fed newborns. Rosen and Lawrence (1994) were among the earliest to look at newborn feeding in relation to labor medication in both breast- and bottle-fed newborns in the immediate postpartum period. They used newborn weight gain as a measurement for successful feeding and concluded that epidural analgesia did not have any appreciable impact on newborn weight loss in the first days of life.

Halpern and associates (1999) used duration of breastfeeding to evaluate the effect of pain medication given to the mother in labor on successful feeding behaviors. They interviewed 171 women 6 to 8 weeks postpartum who had received either parenteral narcotics, epidural analgesia, or no medication for Labor pain relief. They could not demonstrate a correlation between breastfeeding success rates and the use of epidural analgesia, local anesthetics, or opioids for labor analgesia and concluded that pain medication given to mothers in labor did not affect 
breastfeeding success. This study also reported that $36 \%$ of the participants experienced breastfeeding problems but did not provide results related to whether the problems were associated with use of analgesia during labor.

In contrast, Riordan, Gross, Angeron, Krumwiede, and Melin (2000) reported lower breastfeeding assessment scores in newborns whose mothers received either intravenous, epidural, or a combination of intravenous and epidural pain medication during labor compared with mothers who received no pain medication. This study did not take into account other variables known to alter newborn sucking postbirth, such as timing of first breastfeeding, mother-newborn proximity, neonatal events such as vacuum or forceps deliveries or deep oral suctioning, and did not measure the amount of epidural drug to which the neonate was exposed during the labor process.

These studies are difficult to evaluate because of inconsistencies in design, study population, anesthesia techniques used, timing of epidural initiation in relation to the labor curve, differences in drugs used, and differences in drug dosage and administration procedures. Given these considerations, the aim of this study was to investigate whether epidural analgesia using ultra low dose fentanyl and bupivacaine affected the initiation of breastfeeding behaviors in healthy full-term newborns.

\section{Method}

The study consisted of two groups. The medicated group included 28 mother-newborn dyads exposed to a bolus of $0.125 \%$ bupivacaine, $50 \mathrm{mcg}$ fentanyl, and concentration of 1:600,000 epinephrine followed by a continuous infusion of $0.044 \%$ bupivacaine, $0.000125 \%$ fentanyl, and concentration of 1:800,000 epinephrine at $14 \mathrm{ml}$ per hour through an epidural catheter during labor. This was the standard protocol developed by the department of obstetric anesthesia specifically for use in laboring women at the study site. The unmedicated group included 28 mother-newborn dyads who were not exposed to any pain medication or central nervous system (CNS) depressants, including local anesthetics for episiotomy repair, during labor.

A labor and delivery nurse recruited participants and obtained their informed consent in the labor and delivery unit. Patients who met the inclusion criteria were approached by a labor and delivery nurse based on a random table of numbers. If the number on the table was an even number, the patient was approached to be in the study. If the number on the table was an odd number, the patient was not approached. Only one patient declined to be in the study, and no patient withdrew after the study began. At delivery, cord blood was obtained for drug concentration analysis of bupivacaine and fentanyl and the newborn was placed immediately on the mother's chest. A blanket was placed over the newborn, who remained in skin-to-skin contact with his or her mother for 1 hour following delivery. The newborn's Apgar scores, vital signs, and assessments were performed while on the mother's chest. All other interventions such as weighing, eye care, vitamin $\mathrm{K}$ administration, and physical examinations were delayed for 1 hour.

When the birth was complete and all evidence of analgesia was out of sight, the data collector was contacted to observe the newborn's breastfeeding behaviors. The data collector was blind as to study group assignment.

The breastfeeding behaviors were recorded using the Preterm Infant Breastfeeding Behavior Scale (PIBBS) (Nygvist, Rubertson, Ewaksm, \& Sjoden, 1996). The assessment included observations of rooting, sucking, and swallowing, the actual number of sucks and sucking bursts, the newborn activity state, the mother's awareness of letdown of breast milk, problems noted by the mothei; and any environmental influences. The PIBBS was initially designed for use with premature newborns to evaluate their maturational progress in establishing breastfeeding behaviors. The tool tested equally well with full-term neonates (Nygvist et al., 1996) and was chosen for this study because it contained behaviors related to latch on as well as other feeding behaviors. The PIBBS better defined the behaviors than did other available instruments and also considered observations of the mother.

At approximately 1 hour of age, the data collector measured neurobehavior in the newborn using the Neurologic and Adaptive Capacity Score (NACS) (AmielTison et al., 1982). The evaluated behaviors included response and habituation to light and sound, passive tone, active tone, primary reflexes, and a general assessment. The newborns then received routine nursery care and were returned to their mothers. All of the newborns remained with their mothers, unless the mother specifically asked for the newborn to be returned to the nursery, and were breastfed on demand for the next 24 hours. At approximately 24 hours of age, the newborns were again evaluated with the PIBBS and NACS. Permission to use the PIBBS and NACS was obtained prior to the start of the study. The newborns were weighed before and after feedings. None of the newborns received supplemental formula or a pacifier during the course of the study.

After the conclusion of the study, the medical records of the participants were reviewed. Information related to the participants' current and previous breastfeeding experience, labor and delivery history, and the newborns' assessments were recorded.

\section{Participants}

The mothers in the study were at least 18 years of age, had given birth to at least one previously viable newborn, received no pain medication during labor except for the drugs under study, had a normal vaginal delivery, and were well enough postpartum to require no more than the 
standard routine care. The newborns were normal and full-term, with Apgar scores of at least 8 at 1 and 5 minutes, and had no evidence of in utero or birth asphyxia. The newborns all were considered to be healthy enough to room-in with their mothers and to require no more than standard newborn care.

All mothers and newborns were discharged home within 48 hours of delivery without complications. All newborns were exclusively breastfeeding at the time of hospital discharge.

\section{Site}

The study was conducted at a large intercity tertiary medical center and its community-based affiliate hospital. Because all of the participants who received epidural analgesia were from the tertiary medical center, they received the same epidural infusion dosage and rate of administration. All other aspects of care were similar between the two institutions. Physicians caring for the women had privileges at both institutions, and nurses were oriented to both institutions. Both hospitals followed the same policies and procedures.

\section{Results}

Data were obtained from the cord blood of the 56 mother-newborn dyads and during 112 breastfeeding sessions and 112 neurological examinations of the newborns. A type I error of .05 was used for all tests of statistical significance. Data were analyzed using the SPSS PC computer program.

\section{Lack of successful breastfeeding was more attributable to maternal fatigue, anxiety, lack of breastfeeding experience, induction of labor, and extremes of the labor curve than to labor analgesia.}

All data were obtained by the principal investigator, who stayed with the mother-newborn dyad during the data collection sessions and recorded and timed all observed behaviors. The data were then coded and examined graphically in box plots and within group histograms to see if the distribution of variables in each group was symmetrical and free of gross outliers and if the spread of data across groups was fairly constant. Most variables tended to follow a normal distribution, but several were severely skewed. The skewed variables were areolar grasp, environment, problems, rooting on the PIBBS, and general assessment on the NACS. The reason for the skewness was that the scores demonstrated almost no variability between the participants. The skewed variables were not included in the final analysis (conducted with and without the skewed variables, with no difference in results).

Measures of central tendency were calculated for each group. Changes in the mean values for the variables were calculated, and comparisons between groups were made using analysis of variance. Based on the data, analysis of variance for repeated measures was used to analyze the difference between groups over time as well as the differences between individual participants over time. Measures of central tendency were calculated for the variables maternal age, gravida, para, length of stage I labor, length of stage II labor, newborn birth weight, use of Pitocin (Parkedale Pharmaceuticals, Inc, Rochester, Ml) (oxytocin), previous breastfeeding experience, number of times newborn fed in 24 hours, and newborn sex to evaluate for homogeneity of the sample. A $\boldsymbol{t}$ test was performed on interval-level variables and a chi-square test on nominal-level variables to determine if the difference in mean score was significant between the medicated and unmedicated groups.

The women in both groups were similar in respect to gravidity (mean unmedicated $=3$; mean medicated $=3.5$; $p=.2$ ), parity (mean unmedicated $=1.8$; mean medicated $=$ $1.7 ; \mathrm{p}=.7$ ), previous breastfeeding experience (unmedicated $=79 \% ;$ medicated $=82 \% ; p=.7$ ), and the birth weight of their newborns (mean unmedicated $=3,396$ g; mean medicated $=3,483 \mathrm{~g} ; \mathrm{p}=.5$ ). There was a slight difference in the mean time for both stage I (mean unmedicated $=5.2$ hours, mean medicated $=6.5$ hours; $p=.2$ ) and stage II (mean unmedicated = 15 minutes; mean medicated= 20 minutes; $p=.4$ ) of labor between the groups. The mothers in the medicated group tended to feed their newborns more often, but these differences were not clinically significant (mean unmedicated $=4.1$; mean medicated $=5.4 ; \mathrm{p}=.07)$.

There were significant differences in maternal age, use of Pitocin, and sex of the newborn between the two groups. Women in the medicated group tended to be slightly older, with a mean age of 31 years (SD = 4.7), compared with 27.5 years $(S D=5.28)$ in the unmedicated group, and were more likely to receive Pitocin to induce or augment their labor than women in the unmedicated group (unmedicated $=32 \%$, medicated $72 \% ; p=$ $.003)$. There were also more male newborns in the medicated group than in the unmedicated group (unmedicated $=39 \%$, medicated $=68 \% ; p=.03$ ).

All but six newborns studied demonstrated breastfeeding behaviors in the 1st hour postbirth. The initial breastfeeding behaviors, which included rooting, searching for the breast, and attempting to latch onto the breast, began as early as 6 minutes of life and as late as 40 minutes of life, with a mean of 24 minutes. There was no statistical- 


\section{TABLE 1}

Measures of Central Tendency and ANOVA for the Initial Breastfeeding Behaviors Using the PIBBS (n = 28 immedicated; 28 medicated)

\begin{tabular}{|c|c|c|c|c|c|}
\hline Variable & Range & Mean & $\begin{array}{l}\text { Standard } \\
\text { Deviation }\end{array}$ & $\mathbf{F}$ & $\mathbf{p}$ \\
\hline \multicolumn{6}{|c|}{ Newborn behavior } \\
\hline Unmedicated & $1-8$ & 4.5 & 1.73 & 0.450 & $n s$ \\
\hline Medicated & $1-8$ & 4.2 & 1.85 & & \\
\hline \multicolumn{6}{|c|}{ Time newborn held/minutes } \\
\hline Unmedicated & $10-60$ & 37.61 & 14.28 & 2.322 & $n s$ \\
\hline Medicated & $10-60$ & 31.18 & 17.18 & & \\
\hline \multicolumn{6}{|c|}{ Time latched on/minutes } \\
\hline Unmedicated & $5-60$ & 16.67 & 11.99 & .001 & $n s$ \\
\hline Medicated & $1-50$ & 16.78 & 14.65 & & \\
\hline \multicolumn{6}{|c|}{ Longest sucking burst (\# of sucks) } \\
\hline Unmedicated & $5-43$ & 19.39 & 8.9 & 2.802 & $n s$ \\
\hline Medicated & $0-42$ & 14.89 & 11.0 & & \\
\hline \multicolumn{6}{|l|}{ Sucking } \\
\hline Unmedicated & $2-4$ & 3.03 & 0.5 & 1.704 & ns \\
\hline Medicated & $0-4$ & 2.75 & 1.5 & & \\
\hline \multicolumn{6}{|l|}{ Swallowing } \\
\hline Unmedicated & $0-2$ & 0.92 & 0.46 & 4.036 & ns \\
\hline Medicated & $0-1$ & 0.64 & 0.48 & & \\
\hline
\end{tabular}

ly significant difference between the means of the medicated and unmedicated groups $(F=.293 ; p=n s)$ in relation to the amount of time it took for the newborns to initiate breastfeeding behaviors after birth.

Data collected during the initial breastfeeding session after birth, using the PIBBS, were analyzed using ANOVA to determine whether there were any statistically significant differences between the groups. There were slight differences in the mean scores for the amount of time the newborn was held, the number of sucks composing the longest sucking burst, and the frequency of swallowing. Mothers in the unmedicated group tended to hold their newborns 6 minutes longer and to have newborns whose longest sucking burst was five sucks greater and who occasionally swallowed. These differences were not statistically significant. The results are contained in Table 1 .

A comparison of the groups, from the initial breastfeeding session to one at 24 hours, showed a slight difference in the time latched on and the longest sucking burst. Newborns in the unmedicated group tended to remain latched onto the breast 2 minutes longer. The number of sucks in their longest sucking burst was four sucks greater than newborns in the medicated group. These differences were not statistically significant. These results are contained in Table 2.

Although these results did not demonstrate significant differences in breastfeeding behaviors between the med- icated and unmedicated newborns, several newborns scored significantly below the mean on the PIBBS. These newborns were further evaluated to determine if there were any identifiable factors that were common among them. Six newborns scored below the mean, three in the medicated group and three in the unmedicated group. All six newborns scored low immediately postbirth and at 24 hours of age. Compared with the full sample, these six newborns had a higher incidence of mothers who had not previously breastfed, complained of unusual fatigue postdelivery, were induced for labor, or experienced extremes of the labor curve.

The results of the NACS demonstrated statistically significant differences in the variables primary reflexes $(F=$ 4.928; $p=.03)$ and adaptive scores $(F=4.029 ; p=.05)$ on the initial examination after birth and in primary reflexes $(F=5.586 ; p=.02)$ on the second examination at 24 hours of age. In addition, data were analyzed to determine if differences existed between the groups from the initial examination to the second examination. Repeated measures ANOVA was used to analyze differences between the groups over time.

The analysis indicated that there were statistically significant differences in the scores between the first and second examination of the same newborn but not between the medicated and unmedicated groups. The newborns' scores consistently increased from the first to second 
TABLE 2

Measures of Central Tendency and ANOVA for Breastfeeding Behaviors at 24 Hours of Age Using the PIBBS ( 1 = 28 , mmedicated; 28 medicated)

\begin{tabular}{|c|c|c|c|c|c|}
\hline Variable & Range & Mean & $\begin{array}{l}\text { Standard } \\
\text { Deviation }\end{array}$ & $\mathbf{F}$ & p \\
\hline \multicolumn{6}{|c|}{ Newborn behavior } \\
\hline Unmedicated & $1-8$ & 4.28 & 2.01 & 0.808 & $n s$ \\
\hline Medicated & $1-8$ & 3.71 & 2.69 & & \\
\hline \multicolumn{6}{|c|}{ Time newborn held/minutes } \\
\hline Unmedicated & 7-75 & 35.67 & 15.45 & 0.059 & $n s$ \\
\hline Medicated & $10-60$ & 36.75 & 17.36 & & \\
\hline \multicolumn{6}{|c|}{ Time latched on/minutes } \\
\hline Unmedicated & $3-50$ & 14.85 & 9.83 & 0.943 & $n s$ \\
\hline Medicated & $1-50$ & 12.21 & 10.52 & & \\
\hline \multicolumn{6}{|c|}{ Longest sucking burst (\# of sucks) } \\
\hline Unmedicated & $0-29$ & 16.82 & 6.5 & 3.038 & .087 \\
\hline Medicated & $0-48$ & 12.89 & 9.9 & & \\
\hline \multicolumn{6}{|l|}{ Sucking } \\
\hline Unmedicated & $1-4$ & 3.03 & 0.5 & 0.199 & ns \\
\hline Medicated & $1-4$ & 2.71 & 0.9 & & \\
\hline \multicolumn{6}{|l|}{ Swallowing } \\
\hline Unmedicated & $0-2$ & 0.89 & 0.42 & 0.861 & $n s$ \\
\hline Medicated & $0-3$ & 0.75 & 0.70 & & \\
\hline \multicolumn{6}{|c|}{ Weight gain/milligrams } \\
\hline Unmedicated & $0-10$ & 2.43 & 2.44 & 0.091 & ns \\
\hline Medicated & $0-16$ & 2.67 & 3.63 & & \\
\hline
\end{tabular}

examination. The variable primary reflexes was the only variable for which the score remained low in the medicated group at the second examination at 24 hours of age. The results are contained in Table 3 .

Analysis of the cord blood for drug concentration levels of bupivacaine and fentanyl showed that the levels detectable at the time of delivery were low in relation to the dose infused. The assay used for this study was able to detect drug presence at the nanogram level. The levels of bupivacaine ranged from $0.1 \mathrm{ng}$ per milliliter to $98.6 \mathrm{ng}$ per milliliter, with a mean of $31.81 \mathrm{ng}$ per milliliter and a standard deviation of 22.54. The level of bupivacaine was related, however, to the length of time the epidural was infusing. The longer the epidural was in place, the greater the concentration of bupivacaine in the cord blood at delivery. When the levels were assessed using regression analysis, the results were significant for the amount of bupivacaine and the length of time the epidural was infused $(\mathbf{R}=.42, F=17.631 ; p=.0001)$.

The levels of fentanyl ranged from nondetectable to $0.42 \mathrm{ng}$ per milliliter, with a mathematical mean of $0.0042 \mathrm{ng}$ per milliliter and a standard deviation of $0.0083 \mathrm{ng}$ per milliliter. Fentanyl was not detectable with the assay used in this study below the levels of $0.01 \mathrm{ng}$ per milliliter. When assessed using regression analysis, the levels of fentanyl and the amount of time the drug was infused were not statistically significant $(\mathrm{R}=.04 ; F=$ 0.956; $p=\mathbf{n s ) . ~}$

The levels of bupivacaine were significant in relation to the newbom's passive tone $(\mathrm{F}=4.249 ; p=.05)$, active tone $(\mathrm{F}=9.34 ; p=.005)$, and total score on the N A C S (F = 4.499; $p=.04$ ) during the initial examination but not during the second examination at 24 hours of life. There were no significant findings between the levels of bupivacaine and any of the PIBBS variables. There were also no significant findings between fentanyl and the NACS or PIBBS variables. This suggests that the drug effects identified in this population were primarily due to bupivacaine.

A regression analysis was performed on the PIBBS variables to determine if the score obtained had any relationship to the amount of weight change the newborn experienced during the feeding. It was expected that as the score of the PIBBS increased, the newborn would be demonstrating more efficient breastfeeding behaviors and this would result in greater milk transfer. Because the newborn was weighed immediately before and after breastfeeding, the weight change was attributed to the volume of milk ingested during that feeding. Results of the analy- 
TABLE 3

Repeated Measures AIXOVA for the NACS $(n=28$ unmedicated; 28 medicated)

\begin{tabular}{|c|c|c|}
\hline Variable & $\mathbf{F}$ & p \\
\hline \multicolumn{3}{|c|}{ Active tone } \\
\hline Time $^{3}$ & 31.670 & .0001 \\
\hline Group $^{b}$ & 0.695 & nS \\
\hline \multicolumn{3}{|l|}{ Adaptive } \\
\hline Time & 11.251 & .001 \\
\hline Group & 0.868 & $n s$ \\
\hline \multicolumn{3}{|c|}{ Passive tone } \\
\hline TIIIle & 27.286 & .0001 \\
\hline Group & 1.016 & ns \\
\hline \multicolumn{3}{|c|}{ Primary reflexes } \\
\hline Time & 14.370 & .0001 \\
\hline Group & 1.243 & ns \\
\hline \multicolumn{3}{|l|}{ Total score } \\
\hline Time & 78.984 & .0001 \\
\hline Group & 0.763 & ns \\
\hline \multicolumn{3}{|c|}{$\begin{array}{l}{ }^{3} \text { Time refers to the difference in scores from the initial examination } \\
\text { to the examination at } 24 \text { hours. }\end{array}$} \\
\hline \multicolumn{3}{|c|}{$\begin{array}{l}\text { bGro11p refers to the difference between the scores of the medicated } \\
\text { and unmedicated groups. }\end{array}$} \\
\hline
\end{tabular}

sis were statistically significant $(\mathrm{R}=.746 ; F=23.936 ; p=$ .0001) for predicting weight gain from the PIBBS score.

\section{Discussion}

The purpose of this study was to investigate whether differences existed between the breastfeeding behaviors of neonates born to mothers who were unrnedicated and those who received epidural analgesia during labor and whether these differences were related to central nervous system depression in the newborn. This study did not find any significant differences in the breastfeeding behaviors of the newborns in the two groups. The findings did support the existence of a relationship between a newborn's exposure to epidural analgesia during labor and certain neurobehaviors exhibited postbirth.

There could be many reasons for the results obtained in this study. The most important of these is that epidural analgesia has no effect on the breastfeeding behaviors of normal full-term neonates or that the effect is so small that it is not detectable by the instruments used in this study. Another consideration is that the amount of drug the newborn was exposed to was not sufficient to cause detectable differences in this population. An additional possibility is that the participants were exposed to conditions that were supportive of breastfeeding and they were able to overcome any effects of the analgesia. In addition, differences between the unrnedicated and medicated groups could also be contributing to the lack of significant findings. Newborns in the unmedicated group could have been exposed to stressors that were different from those experienced by newborns in the medicated group. Those stressors could be as significant as those experienced by newborns in the medicated group.

The amount of drug used in this study population was ultra low, compared with standard infusions. The dosages reported in the literature in the 1990s for labor analgesia ranged from $0.25 \%$ to $0.75 \%$ solutions of bupivacaine, with infusion concentrations ranging from 1 to $5 \mathrm{mg}$ per milliliter administered between 5 and $35 \mathrm{ml}$ per hour. Dosages of fentanyl ranged from 0.005 to $0.025 \mathrm{mg}$ per milliliter, with infusion rates of 0.2 to $1.5 \mathrm{mg}$ per hour (Bonica \& MacDonald, 1995; Palmer, VanMaren, Nogami, \& Alvesm, 1999). These medications were administered separately or in combination and as a bolus, continuous infusion, or a combination of the two.

The amount of drug detectable in neonates' blood or the cord blood at delivery was reported in only a few studies, however. Bader and associates (1995) found the fentanyl concentrations in maternal blood at the time of delivery to be almost identical to the concentration found in the cord blood. Because the dose used in the current study was $0.044 \%$ bupivacaine and $0.000125 \%$ fentanyl at a continuous infusion of $14 \mathrm{ml}$ per hour $(0.6 \mathrm{mg}$ bupivacaine and $0.00175 \mathrm{mg}$ fentanyl per hour), the patients were receiving a dose lower than is typically reported for labor analgesia. This may account for the lack of detectable effect on the newborns in this study. Both

\section{There were no measurable differences in the}

\author{
breastfeeding behaviors of neonates born to \\ mothers who received ultra low dose epidural \\ analgesia for labor and of neonates whose \\ mothers were unmedicated.
}

bupivacaine and fentanyl cross the placenta readily, and it is not surprising that smaller dosages would have less effect.

It does not appear that fentanyl has a cumulative effect over time (Bader et al., 1995). In the classic study examining the amount of fentanyl in maternal blood compared with that found in cord blood, the results between the mother and fetus were almost identical (Bader et al., 1995). In addition, concentrations of fentanyl in cord blood were very low compared with the amount infused. Maternal blood studies of fentanyl concentrations indi- 
cate that there is a rapid decline of detectable drug within 1 to 60 minutes after a bolus (Bader et al., 1995). This decline implies rapid redistribution and uptake by maternal tissues. What happens in the fetus is unknown, but when in vitro human placental models were studied in relation to the transfer of fentanyl, a plateau was reached within 40 minutes of the initiation of a continuous infusion (Zakowski, Schlesinger, \& Dumbroff, 1993) and levels did not increase after that time. Therefore, the length of time the woman was exposed to the drug during labor was not as significant as the actual dosage infused. The findings of this study also indicate that the length of time that the fetus was exposed to the infusion of fentanyl during labor had no effect on the overall outcome.

It is interesting to speculate that if blood concentrations of bupivacaine and fentanyl are low, this may be in part due to uptake by fetal tissues. The significance of fetal uptake of bupivacaine is unknown. It has been sug-

\section{It is possible that women can successfully establish breastfeeding and experience a pain-free labor if the analgesia is carefully chosen and administered.}

gested (Bader et al., 1995) that the uptake of bupivacaine by nonvital organs of the fetus may actually prevent higher accumulation in the fetal brain and myocardium. There is some evidence that the newborn is able to metabolize and excrete bupivacaine in a similar manner as an adult (Kuhnert et al., 1985), which would assist in preventing accumulation. Detectable amounts are found in the newhorn's urine for approximately 36 hours after birth. In addition, the absence of significant neurobehavioral effects in the neonate suggests that significant central nervous system accumulation is unlikely, provided the dosage has remained low.

As the exposure increases, however, reported neurological alterations become more apparent. The initial studies of bupivacaine reported that neonates were more likely to be cyanotic and unresponsive to their surroundings (Rosenblatt et al., 1981). Visual skills and alertness decreased significantly as levels of bupivacaine increased. In contrast, it appears that as the dose of bupivacaine is reduced, neurobehavioral changes in the newborn become more difficult to detect.

The question as to whether the PIBBS was sensitive enough to detect differences in breastfeeding behaviors, which might affect the results of this study, also was considered. The sensitivity of the instrument, however, was established when it was demonstrated that it could pre- diet which newborns were exhibiting behaviors sufficient to effect weight change during the feeding. If the newborn scored 3 or higher on the variable sucking, 2 on the variable swallowing, and 10 or higher on the variable longest sucking burst, a weight gain of 1 to $3 \mathrm{~g}$ was observed during the feeding. In addition, if the newborn scored 20 or greater on the variable latch, a weight change of 2.5 to 5 mg was observed.

The individual differences of the participants also were considered in relation to the results obtained. Although this study did not demonstrate significant differences between the two groups, 6 study neonates had considerable difficulty breastfeeding. In this small subpopulation, 3 of the mothers had no previous breastfeeding experience. Two of the newborns who had trouble breastfeeding were born to mothers who delivered by natural childbirth but were so exhausted following delivery that they fell asleep during the recovery period and could not be aroused to feed their newborns. Both of these mothers were experienced in breastfeeding. One newborn did not feed well due to environmental factors. This mother attempted to feed her newborn in the presence of her four other children, all under the age of 5 . This same newborn scored low on the PIBBS at the initial breastfeeding session after birth, in which there were no distractions, but the mother did complain of extreme fatigue.

Compared with the full sample, this subsample had a higher incidence of women without previous breastfeeding experience, fatigue, induction of labor, or extremes of the labor curve. Analysis of the data with these patients removed from the sample did not affect the results of the study but may be important in the clinical assessment of the mother-newborn breastfeeding dyad.

\section{Conclusions}

The data collected from this study did not show any measurable differences between the breastfeeding behaviors of neonates born to mothers who received epidural analgesia for labor and those whose mothers were unmedicated. It is unlikely that the individual differences in participants affected the results. Although the newborns' differences may have had an impact on the breastfeeding behaviors demonstrated, these differences were distributed equally between both groups and did not affect the results of the overall study.

A factor that may have influenced the results was the ultra low concentration of bupivacaine and fentanyl experienced by the sampie in this study. For many years, the breastfeeding literature has implied that pain medication given to the mother in labor may have a negative impact on her newborn's ability to breastfeed afterward (Righard \& Alade, 1992). Although there is evidence that exposure to high doses of narcotics causes central nervous system depression in the neonate that can last for several days 
(Kuhnert et al., 1985), there is not sufficient evidence that ultra low dose analgesia has a similar effect.

Health care professionals should be cautious when providing information to their patients about potential barriers to breastfeeding success. Many mothers fear the effect medications may have on their fetus or newborn. It is unknown how many decline to breastfeed or feel overly anxious about their baby's ability to breastfeed because of medications they took in labor and are now powerless to control. A mother's desire for pain relief during labor may be greater than her desire to breastfeed at that point in time. If she believes that the decisions she made in labor are negatively affecting her newborn, she may become more anxious during the feeding, and that anxiety may affect her ability to successfully breastfeed.

The benefits of breastfeeding are sufficiently strong that health care professionals should encourage and support this method of feeding in all but the most adverse circumstances. The results of this study indicate that the use of ultra low dose analgesia in labor does not pose a barrier to successful breastfeeding in healthy full-term neonates. This study would need to be repeated, however, using a larger sample and various combinations of medications and dosages, before any general statement could be made about the relationship between epidural analgesia and breastfeeding. At least in this limited population, counseling the mother to avoid epidural analgesia because it may negatively affect her breastfeeding success may not be in the best interest of the mother-newborn dyad. Pain relief in labor has always been a high priority for childbearing women. This priority may be so high that she may be willing to sacrifice breastfeeding to achieve it. It is possible for women to breastfeed successfully and to experience a pain-free labor if the analgesia is carefully chosen and administered.

\section{REFERENCES}

Abboud, T., Khoo, S., Miller, F., Doan, T., \& Henriksen, E. (1982). Maternal, fetal, and neonatal responses after epidural analgesia with bupivacaine, 2 chloroprocaine, or lidocaine. Anesthesia, 61, 638-644.

Amano, K., Nishijima, M., \& Arai, M. (1985). Effects of local anesthetics on fetal heart rate patterns and neonatal neurobehavior. Acta Obstetrica et Gynaecologica Japonica, 37(11), 2291-2299.

Amiel-Tison, C., Barrier, G., \& Shnider, S. (1982). A new neurologic adaptive capacity scoring system for evaluating obstetric medication in full term infants. Anesthesiolo ${ }_{g y}$, $56,340-347$.

Bader, A., Fragneto, R., Terui, K., Arthur, R., Loferski, B., \& Datta, S. (1995). Maternal and neonatal fentanyl and bupivacaine concentrations after epidural infusions during labor. Anesthesia and Analgesia, 81, 829-832.

Belsey, E., Rosenblatt, D., Lieberman, B., Redshaw, M., Caldwell, J., Notarianni, L., et al. (1981). The influence of maternal analgesia on neonatal behavior. British Journal of Obstetrics \& Gynecolo Gy $_{y}, 88(4), 398-406$.

Blackbill, Y., Kane,]., Manniello, R., \& Abramson, D. (1974). obstetric premedication and infant outcomes. American Journal of Obstetrics and Gynecolo ${ }_{g y}, 118,377-384$.

Bonica, J., \& MacDonald, J. (1995). Obstetric analgesia \& anesthesia. Baltimore: Williams \& Wilkins.

Brazelton, T. (1961). Effects of maternal medication on the neonate and his behavior. Pediatrics, 58, 513-518.

Carrie, L., O'Sullivan, G., \& Seegobin, R. (1981). Epidural fentanyl in labour. Anaesthesia, 36, 965-969.

Chapman,]., Macey, M., Keegan, M., Borum, P., \& Bennett, S. (1985). Concerns of breast-feeding mothers from birth to 4 months. Nursing Research, 36(6), 374-377.

Corke, B. (1977). Neurobehavioral responses of the newborn: The effect of different forms of maternal analgesia. Anesthesia, 32, 539.

Crowell, M., Hill, P., \& Humenick, S. (1994). Relationship between obstetric analgesia and time of effective breast feeding. Journal of Nurse Midwifery, 39(3), 150-156.

Eddleston, J., Maresh, M., Horseman, E., Young, H., Lacy, P., \& Anderson, J. (1992). Comparison of the maternal and fetal effects associated with intermittent or continuous infusion of extradura1 analgesia. British Journal of Anesthesia, 69, 154-158.

Frantz, K., \& Fleiss, P. (1980). Ineffective sucking as a frequent cause of failure to thrive in a totally breastfed infant. In $\mathrm{S}$. Freier \& A. Eidelman (Eds.), Human milk: Its biological and social value. International Symposium on Breastfeeding, Tel Aviv. International Congress Series 518.

Hal $_{\text {p e }}$ rn, S., Levine, T., Wilson, D., MacDonnel, J., Katsiris, S., \& Leighton, B. (1999). Effect of labor analgesia on breastfeeding success. Birth, 26, 83-88.

Hodgkinson, R., Blatt, M., \& Wang, C. (1978). Double-blind comparison of the neurobehavior of neonates following the administration of different doses of $\mathrm{me}_{\mathrm{p}}$ ridine to the mother. Canadian Anesthetists Society Journal, 25(5), 405-411.

Hodgkinson, R., \& Husain, F. (1982). The duration of effect of maternally administered $\mathrm{me}_{\mathrm{p}}$ ridine on neonatal behavior. Anesthesiology, 56, 51-52.

Hoyt, M., \& Youngstrom, P. (1990). Neonatal neurobehavioral effects of continuous epidural fentanyl, bupivacaine and epinephrine in labor. Anesthesiolo $g_{y}, 73(3 \mathrm{~A}), 984$.

Hughes, R., Townsend, P., \& Branum, Q. (1988). Relationship between neonatal behavioral responses and lactation outcomes. Issues in Comprehensive Pediatric Nursing, 11, 271-281.

Jouppila, R., \& Hollmen, A. (1976). The effect of segmental epidural analgesia on maternal and fetal acid-base balance, lactate, serum potassium and creatine phosophokinase during labour. Acta Anesthesiologica Scandinavica, 20, 259-266.

Kangas-Saarela, T., Jouppila, R., \& Jouppila, P. (1987). The effect of segmental epidural analgesia on the neurobehavioral responses of newborn infants. Acta Anesthesiologica Scandinavica, 31, 347-351.

Kileff, M., James, F., Dewan, D., \& Floyd, H. (1984). Neonatal neurobehavioral responses after epidural anesthesia for 
cesarean section using lidocaine and bupivacaine. Anesthesia and Analgesia, 63, 413-417.

Kuhnert, B., Linn, P, \& Kuhnert, P. (1985). Obstetric medication and neonatal behavior. Clinical Perinatology, 12, 423-440.

Loftus, J., Hill, H., \& Cohen, S. (1995). Placental transfer and neonatal effects of epidural sufentanil and fentanyl administered with bupivacaine during labor. Anesthesiology, 83, 300-308.

Lothian, J. (1995). It takes two to breastfeed: The baby's role in successful breastfeeding. journal of Nurse Midwifery, 40, 328-334.

Murray, A., Dolby, R., Nation, R., \& Thomas, D. (1981). Effects of epidural analgesia on newborns and their mothers. Child Development, 82, 71-82.

Nygvist, K., Rubertson, C., Ewaksm, U., \& Sjoden, P. (1996). Development of the pretenn infant breastfeeding behavior scale (PIBBS). A study of nurse-mother agreement. journal of Human Lactation, 12(3), 207-219.

Okutorni, T., Mochizuki, J., Asmano, K., \& Hoka, S. (2000). Effect of epidural epinephrine infusion with bupivacaine on labor pain and mother-fetus outcome in humans. Regional Anesthesia and Pain Medication, 25(3), 228234.

O'Le ${ }_{\text {a r y }}$ A., Kopsell, D., \& Haller, S. (1997). Breastfeeding incidence after early discharge and factors influencing breastfeeding cessation. Journal of Obstetric, Gynecologic, and Neonatal Nursing, 26, 289-294.

Palmer, V., VanMaren, G., Nogami, W., \& Alvesm D. (1999). Bupivacaine augments intrathecal fentanyl for labor analgesia. Anesthesiolo ${ }_{g}$, 91, 1-16.

Righard, L., \& Alade, M. (1992). Sucking technique and its success in breastfeeding. Birth, 19, 185-189.

Riordan, J., Gross, A., Angeron, J., Krumwiede, B., \& Melin, J. (2000). The effect of labor pain relief medication on neonatal suckling and breastfeeding duration. journal of Human Lactation, 16, 7-12.

Rosen, A., \& Lawrence, R. (1994). The effect of epidural anesthesia on infant feeding. Journal of the University of Rochester Medical Center, 1, 3-7.

Rosenblatt, D., Belsey, W., Lieberman, B., Redshaw, M., Caldwell, J., \& Notarianni, L. (1981). The influence of maternal analgesia on neonatal behavior: Epidural bupivacaine. British journal of Obstetrics \& Gynecology, 88, 407-413.
Sapkoski, C., Lester, B., Ostheimer, G., \& Brazelton, T. (1992). The effect of maternal anesthesia on neonatal behavior during the first month. Development and Medical Child Neurology, 34(2), 1072-1080.

Scanlon, J., Ostheimer, G., Lurie, A., \& Briwb, W. (1976). Neurobehavioral responses of newborn infants after maternal epidural anesthesia. Anesthesiology, 40, 121-128.

Scherer, R., \& Holzgreve, W. (1995). Influence of epidural analgesia on fetal and neonatal well-being. European Journal of Obstetrics, Gynecology \& Reproductive Biology, 59, 517-529.

Swanstrom, S., \& Bratteby, L. (1981). Metabolic effect of obstetric regional anesthesia and of asphyxia in the newborn infant during the first two hours after birth: ill: Adjustment of arterial blood gases and acid-base balance. Acta Paediatrica Scandinavica, 70, 811-818.

Tronick, E., Wise, S., \& Als, H. (1976). Regional obstetric anesthesia and newborn behavior: Effect over the first ten days of life. Pediatrics, 58, 94-100.

Vandiver, T. (1997). Relationship of mothers' perceptions and behaviors to the duration of breastfeeding. Psychological Reports, 80(3), 1375-1384.

Walker, M. (1997). Do labor medications affect breastfeeding? journal of Human Lactation, 13(2), 131-137.

Wiener, P, Hogg, M., \& Rosen, M. (1979). Neonatal respiration, feeding, and neurobehavioral state. Anaesthesia, 34, 996-1004.

Zador, G., \& Nilsson, B. (1974). Low dose intermittent epidural anaesthesia with lidocaine for vaginal delivery: I. Influence on labour and fetal acid-base status. Acta Obstetricia et Gynecologica Scandinavica, 34, 17-30.

Zakowski, M., Schlesinger, J., \& Dumbroff, S. (1993). In vitro human placental uptake and transfer of fentanyl. Anesthesiology, 79, 1006.

Sharo11 $\operatorname{Rad}_{z y}$ minski, PhD, RN, is all assistant professor and graduate program director, Departme11t of Nursing, Cleveland State University, Cleveland, $\mathrm{OH}$.

Address for correspondence: Sharon $\operatorname{Rad}_{z y}$ millski, PhD, RN, Cleveland State University, Department of Nursing, Rhodes Tower, 1983 East 24th Street, Cleveland, OH 44115. E-mail: s.radzyiltsi@csuohio.edu. 